einstein

Publicação Oficial do Instituto Israelita

de Ensino e Pesquisa Albert Einstein

ISSN: 1679-4508 | e-ISSN: 2317-6385

Adequação às faixas etárias pediátricas de medicamentos novos registrados no Brasil de 2003 a 2013

\section{Suitability of new drugs registered in Brazil from 2003 to 2013 for pediatric age groups}

\author{
Jaqueline Cristina da Silveira Xavier e Castro', Stephanie Ferreira Botelho', \\ Taisa Roberta Lopes Machado', Maria Auxiliadora Parreiras Martins"1, \\ Liliana Batista Vieira², Adriano Max Moreira Reis ${ }^{1}$ \\ 1 Faculdade de Farmácia, Universidade Federal de Minas Gerais, Belo Horizonte, MG, Brasil. \\ 2 Faculdade de Ciências Farmacêuticas, Universidade Federal de Alfenas, Alfenas, MG, Brasil.
}

DOI: 10.31744/einstein journal/2018A04354

\section{RESUMO}

Objetivo: Analisar a adequação às faixas etárias pediátricas dos medicamentos novos registrados no Brasil no período de 2003 a 2013. Métodos: Estudo descritivo dos medicamentos com indicação pediátrica incluídos em uma coorte retrospectiva de medicamentos novos registrados no Brasil. A avaliação da adequação do medicamento à faixa etária pediátrica foi realizada empregando os seguintes critérios: adequação da forma farmacêutica e capacidade de fornecer a dose recomendada. Os medicamentos foram considerados adequados às faixas etárias pediátricas quando preencheram os dois critérios. A análise estatística compreendeu cálculo de frequências e proporções. Resultados: A adequação devido à capacidade do medicamento fornecer a dose recomendada foi superior a $80 \%$ em todas as faixas etárias. Em relação à adequação da forma farmacêutica, identificou-se que quanto maior a faixa etária, maior a proporção de adequação para uso pediátrico. Em relação aos medicamentos que se apresentavam em formas farmacêuticas sólidas, evidenciou-se que metade foi classificada como inadequada para uma ou mais faixas etárias pediátricas para as quais estavam indicados. A adequação dos medicamentos à faixa etária pediátrica foi $64,3 \%$ para pré-escolares, $66,7 \%$ para recém-nascidos a termo, $66,7 \%$ para recém-nascidos prematuros e superior a $70 \%$ para as demais faixas etárias. Conclusão: 0s medicamentos destinados às crianças menores de 6 anos apresentaram menor frequência de adequação, considerando a forma farmacêutica e a capacidade de fornecer a dose recomendada. A disponibilidade e a proporção de adequação dos medicamentos para uso pediátrico aumentam com a elevação da faixa etária para a qual o medicamento é registrado.

Descritores: Medicamentos de referência; Aprovação de drogas; Tratamento farmacológico; Formas de dosagem; Criança

\section{ABSTRACT}

Objective: To analyze suitability of new drugs registered in Brazil from 2003 to 2013 for pediatric age groups. Methods: A descriptive study of drugs with pediatric indication included in a retrospective cohort of new drugs registered in Brazil. The evaluation of drug suitability for the pediatric age group was performed using the following criteria: suitability of dosage form and capacity to deliver the recommended dose. The drugs were considered adequate for the pediatric age groups when they met both criteria. The statistical analysis included calculation of frequencies and proportions. Results: Suitability due to the drug capacity to deliver the recommended dose was greater than $80 \%$ across all age groups. Regarding suitability of the dosage form, we identified that the older the age group, the greater suitability for pediatric use. Concerning the drugs presented in solid 
dosage form, we showed that half were classified as inadequate for one or more pediatric age groups to whom they were indicated. The adequacy of drugs to the pediatric age group was $64.3 \%$ for preschool children, $66.7 \%$ for full-term newborns, $66.7 \%$ for premature newborns, and over $70 \%$ for other age groups. Conclusion: Drugs for children aged under 6 years were less often adequate, considering the dosage form and capacity to provide the recommended dose. The availability and proportional suitability of medicines for pediatric use are greater for older age groups, according to age groups the drug is registered for.

Keywords: Reference drugs; Drug approval; Drug therapy; Dosage forms; Child

\section{INTRODUÇÃO}

As crianças não são adultos pequenos, mas um grupo diferenciado e heterogêneo de pacientes, com necessidades específicas em relação aos medicamentos. ${ }^{(1-3)}$ A heterogeneidade é consequência das mudanças que ocorrem na infância em relação ao crescimento e ao desenvolvimento de órgãos e sistemas fisiológicos, que influenciam na farmacocinética e na farmacodinâmica, ${ }^{(1,2)}$ bem como das alterações das habilidades motoras e cognitivas que interferem na administração de medicamentos. ${ }^{(3)}$ Assim, as crianças têm necessidades diferentes dos adultos em termos de dose, forma farmacêutica e habilidade para administração de medicamentos. ${ }^{(1,3,4)}$

Para alcançar a exatidão da dose administrada, reduzir erros de medicação, aumentar a adesão ao tratamento e melhorar os resultados terapêuticos em pediatria, é essencial a disponibilidade de medicamentos em formas farmacêuticas e formulações adequadas às necessidades das crianças. ${ }^{(2)}$

$\mathrm{O}$ desenvolvimento de medicamentos segundo os princípios de uma formulação centrada no paciente ${ }^{(5,6)} \mathrm{e}$ na idade apropriada ${ }^{(4)}$ é uma tendência atual, para atender às especificidades em pediatria. Um medicamento apropriado à criança apresenta forma farmacêutica que propicia liberação variável da dose segundo peso/altura, palatabilidade aceitável, segurança associada a excipientes, facilidade de deglutição, dispositivo adequado para medida da dose e habilidade para administração compatível com a faixa etária pediátrica a que se destina. ${ }^{(4,5,7)}$

Em diferentes países, o número de medicamentos registrados para uso pediátrico é inferior ao de adultos, e a indisponibilidade é maior para medicamentos destinados às crianças mais jovens. ${ }^{(8-11)}$ No Brasil, também há carência de medicamentos adequados ao uso pediátrico. ${ }^{(12)}$

Em virtude do número reduzido de medicamentos adequados às faixas etárias pediátricas, o uso de medicamentos off label ou não licenciado para crianças é um problema que ainda persiste em diferentes cenários da assistência, inclusive no Brasil, elevando o risco de reações adversas e a inefetividade terapêutica. ${ }^{(12)}$

\section{OBJETIVO}

Analisar a adequação das formas farmacêuticas às faixas etárias pediátricas dos medicamentos novos registrados no Brasil no período de 2003 a 2013.

\section{| MÉTODOS}

Trata-se de um estudo descritivo dos medicamentos com indicação pediátrica incluídos em uma coorte retrospectiva de medicamentos novos registrados no Brasil. A coorte retrospectiva abrange os medicamentos novos registrados pela Agência Nacional de Vigilância Sanitária (ANVISA) entre janeiro de 2003 e dezembro de 2013. A coorte foi constituída consultando o banco de dados Drugs@FDA (https://www.accessdata.fda.gov/ scripts/cder/daf/); a seção de publicações da ANVISA disponibilizada no Diário Oficial da União; e o artigo de revisão "To market, to market" publicado no Annual Reports in Medicinal Chemistry. A descrição detalhada da elaboração da coorte foi apresentada em um estudo prévio. ${ }^{(13)}$

Identificaram-se, na lista de preços de medicamentos de janeiro de 2016, publicada pela ANVISA, os medicamentos que estavam ainda em comercialização no Brasil. ${ }^{(14)}$ Consultou-se o bulário eletrônico da ANVISA para verificar se os medicamentos novos incluídos na coorte e em comercialização no país em 2016 tinham registro e indicação pediátrica na ANVISA. ${ }^{(15)}$ Quando não estava disponível no bulário, solicitou-se, por e-mail, a bula ao laboratório fabricante.

A classificação dos medicamentos foi realizada de acordo com o terceiro nível terapêutico farmacológico do Anatomical Therapeutic Chemical Code (ATC), da Organização Mundial da Saúde (OMS). ${ }^{(16)}$

Para caracterização da indicação pediátrica nesta investigação, adotou-se a seguinte estratificação etária: recém-nascido prematuro ( $<37$ semanas), recém-nascido a termo ( $\geq 37$ semanas e $<28$ dias), lactente ( $\geq 28$ dias e $<2$ anos), pré-escolar ( $\geq 2$ e $<6$ anos), escolar ( $\geq 6$ e $<12$ anos) e adolescente ( $\geq 12$ anos e $<19$ anos). ${ }^{(17)}$

A avaliação da adequação do medicamento à faixa etária pediátrica foi realizada considerando as apresentações farmacêuticas comercializadas no Brasil em 2016 e empregaram-se os seguintes critérios: adequação da forma farmacêutica e capacidade de fornecer a dose recomendada. Os medicamentos foram considerados adequados às faixas etárias pediátricas, quando preencheram os dois critérios. 
A avaliação da adequação da forma farmacêutica às faixas etárias pediátricas foi realizada empregando-se os critérios definidos pela European Medicines Agency (EMA) no documento Reflection paper: formulations of choice for the paediatric population. ${ }^{(18)}$ Os critérios foram desenvolvidos empregando um matriciamento dos seguintes fatores: faixa etária estratificada segundo Willians et al., ${ }^{(17)}$ forma farmacêutica e via de administração, considerando a adequação em pediatria. A adequação do medicamento conforme o matriciamento foi definida em uma escala do tipo Likert. Para crianças menores de 11 anos, a escala é estruturada em (1) inadequado, (2) adequado com problemas, (3) adequado mas não preferível, (4) boa adequação e (5) excelente adequação. Para adolescentes, a escala é: (1) inaceitável, (2) aceitável sob reserva, (3) aceitável, (4) preferida e (5) forma farmacêutica de escolha. ${ }^{(18)}$ Nessa investigação, classificou-se a forma farmacêutica como adequada para a faixa etária para a qual o medicamento está registrado quando apresentou pontuação superior a 3, para crianças menores de 12 anos, e superior a 2, para adolescentes.

Avaliou-se também a capacidade de o medicamento, na forma farmacêutica em que é comercializado, fornecer a dose recomendada. Esta avaliação foi realizada segundo Fontan et al., ${ }^{(19)}$ que classifica como inadequação forma farmacêutica sólida - necessidade de divisão para obter a dose adequada - e forma farmacêutica líquida - necessidade de administração de volume do medicamento menor que $1 \mathrm{~mL}$. Todas as demais formas farmacêuticas foram consideradas aptas para fornecer a dose exata necessária à criança. A avaliação de adequação foi realizada considerando a dose prescrita para crianças prevista na bula. Para os medicamentos cuja dose era expressa em $\mathrm{mg} / \mathrm{kg}$, empregaram-se os pesos de referência para cada faixa etária, de acordo com as curvas preconizadas pela OMS para peso ideal de crianças, considerando os limites inferiores da faixa etária. ${ }^{(20)}$

Identificou-se, nas bulas das especialidades farmacêuticas dos 46 medicamentos com indicação pediátrica, a presença dos seguintes excipientes farmacêuticos com potencial de causar dano em crianças: antioxidantes (sulfitos), ${ }^{(21)}$ agentes solubilizantes (polisorbato $80^{(22,23)} \mathrm{e}$ ciclodextrina) ${ }^{(10)}$ conservantes antimicrobianos (parabenos - propilparabeno, etilparabeno e metilparabeno; ${ }^{(22,23)}$ benzoatos - álcool benzílico, ácido benzoico, benzoato de sódio ${ }^{(10,21-23)}$ - e cloreto de benzalcônio), ${ }^{(10,21-23)}$ diluente (lactose), ${ }^{(21,24)}$ edulcorantes (aspartame, ${ }^{(22,23)}$ sorbitol, ${ }^{(10,23)}$ sacarina $^{(10,22)}$ ) e solventes (etanol e propilenoglicol, ${ }^{(10,22-24)}$ e óleo de amendoim). ${ }^{(22)}$

\section{Análise estatística}

O banco de dados foi digitado no programa Epidata 3.1. A análise estatística compreendeu cálculo de frequências e proporções, tendo sido realizada empregando o software Statisical Package for Social Sciences, versão 21.0.

\section{RESULTADOS}

De janeiro de 2003 a dezembro de 2013, foram registrados, no Brasil, 159 medicamentos novos, e 25 interromperam a comercialização no país após o registro. Por isto, a coorte investigada abrangeu 134 medicamentos novos que estavam em comercialização em janeiro de 2016. Entre os 134 medicamentos novos, identificaram-se $46(34,3 \%)$ que tinham indicação pediátrica no exterior e $37(27,6 \%)$ com registro para uso em crianças no Brasil.

Em relação à classificação ATC (Tabela 1) os fármacos mais frequentes pertenciam aos seguintes grupos: (A) trato alimentar e metabolismo $(26,1 \%)$, (J) anti-infecciosos de uso sistêmico $(21,7 \%),(\mathrm{L})$ antineoplásicos e imunomoduladores $(13 \%)$ e $(\mathrm{R})$ sistema respiratório $(10,9 \%)$.

Tabela 1. Anatomical Therapeutic Chemical Code ${ }^{(16)}$ dos 46 medicamentos pediátricos identificados na coorte de medicamentos novos, registrados de 2003 a 2013

\begin{tabular}{|c|c|c|}
\hline \multicolumn{2}{|c|}{ Classificação ATC } & $\mathrm{n}(\%)$ \\
\hline \multicolumn{2}{|c|}{ Trato alimentar e metabolismo } & $12(26,1)$ \\
\hline A02B & $\begin{array}{l}\text { Fármacos para úlcera péptica e doença do refluxo } \\
\text { gastresofágico: dexlansoprazol }\end{array}$ & $1(2,2)$ \\
\hline A04A & Antieméticos e antinauseantes: aprepitanto e palonosetrona & $2(4,4)$ \\
\hline $\mathrm{A} 10 \mathrm{~A}$ & Insulinas e análogos: insulina detemir e insulina glulisina & $2(4,4)$ \\
\hline $\mathrm{A} 16 \mathrm{~A}$ & $\begin{array}{l}\text { Outros produtos que atuam no metabolismo e trato alimentar: } \\
\text { alfa-alglicosidase, alfavelaglicerase, sapropterina, galsulfase, } \\
\text { idursulfase, laronidase e miglustate }\end{array}$ & $7(15,2)$ \\
\hline \multicolumn{2}{|c|}{ Sistema cardiovascular } & $1(1)$ \\
\hline C10A & Agentes modificadores de lipídeos: rosuvastatina & $1(2,2)$ \\
\hline \multicolumn{2}{|c|}{ Anti-infecciosos para uso sistêmico } & $10(21,7)$ \\
\hline J02A & $\begin{array}{l}\text { Antimicóticos para uso sistêmico: anidulafungina, micafungina } \\
\text { e posaconazol }\end{array}$ & $3(6,5)$ \\
\hline J05A & $\begin{array}{l}\text { Antivirais de ação direta: enfuvirtida, entecavir, darunavir, } \\
\text { etravirina, fosamprenavir, raltegravir potássico e atazanavir }\end{array}$ & $7(15,2)$ \\
\hline \multicolumn{2}{|c|}{ Antineoplásicos e agentes imunomoduladores } & $6(13,0)$ \\
\hline L01X & Outros agentes antineoplásicos: nimotuzumabe & $1(2,2)$ \\
\hline L04A & $\begin{array}{l}\text { Imunosupressores: abatacepte, adalimumabe, canaquinumabe, } \\
\text { everolimo e tocilizumabe }\end{array}$ & $5(10,9)$ \\
\hline \multicolumn{2}{|c|}{ Sistema respiratório } & $5(10,9)$ \\
\hline R01A & $\begin{array}{l}\text { Descongestionantes e outras preparações nasais de uso tópico: } \\
\text { ciclesonida e fluticasona }\end{array}$ & $2(4,4)$ \\
\hline R03D & $\begin{array}{l}\text { Outros fármacos sistêmicos para doenças obstrutivas das vias } \\
\text { aéreas: omalizumabe }\end{array}$ & $1(2,1)$ \\
\hline R06A & Anti-histamínicos para uso sistêmico: bilastina e rupatadina & $2(4,4)$ \\
\hline
\end{tabular}


Continuação

Tabela 1. Anatomical Therapeutic Chemical Code ${ }^{(16)}$ dos 46 medicamentos pediátricos identificados na coorte de medicamentos novos, registrados de 2003 a 2013

\begin{tabular}{|c|c|c|}
\hline \multicolumn{2}{|c|}{ Classificação ATC } & n (\%) \\
\hline \multicolumn{2}{|c|}{ Sistema nervoso } & $4(8,8)$ \\
\hline N05A & Antipsicóticos: asenapina e paliperidona & $2(4,4)$ \\
\hline N06A & Antidepressivos: duloxetina & $1(2,2)$ \\
\hline N06B & Psicoestimulantes, agentes usados para TDAH: lisdexanfetamina & $1(2,2)$ \\
\hline \multicolumn{2}{|c|}{ Sistema geniturinário e hormônios } & $2(4,4)$ \\
\hline G03A & $\begin{array}{l}\text { Hormônios anticonceptivos para uso sistêmico: dienogeste; } \\
\text { valerato de estradiol, drospirenona; etinilestradiol }\end{array}$ & $2(4,4)$ \\
\hline \multicolumn{2}{|c|}{ Órgãos dos sentidos } & $2(4,4)$ \\
\hline S01A & Anti-infecciosos: besifloxacino & $1(2,2)$ \\
\hline S01G & Descongestionantes e antialérgicos: alcaftadina & $1(2,2)$ \\
\hline \multicolumn{2}{|c|}{ Dermatológicos } & $1(2,2)$ \\
\hline D06A & Antibióticos para uso tópico: retapamulina & $1(2,2)$ \\
\hline \multicolumn{2}{|c|}{ Sistema musculoesquelético } & $1(2,2)$ \\
\hline $\mathrm{M} 05 \mathrm{~B}$ & $\begin{array}{l}\text { Fármacos que afetam a estrutura óssea e mineralização: } \\
\text { denosumabe }\end{array}$ & $1(2,2)$ \\
\hline \multicolumn{2}{|c|}{ Sangue e órgãos hematopoiéticos } & $1(2,2)$ \\
\hline B02B & Vitamina K e outros hemostáticos: eltrombopag olamina & $1(2,2)$ \\
\hline \multicolumn{2}{|l|}{ Vários } & $1(2,2)$ \\
\hline V03A & Todos os outros produtos terapêuticos: sugamadex & $1(2,2)$ \\
\hline \multicolumn{2}{|l|}{ Total } & $46(100,0)$ \\
\hline
\end{tabular}

Na bula de 40 (87\%) dos medicamentos novos com indicação para crianças constava especificação da faixa etária pediátrica à qual se destinava. Para 6 , dos 46 medicamentos com indicação pediátrica, constava apenas uso pediátrico; estes foram classificados como indicados para todas as faixas etárias, inclusive recém-nascido prematuro e a termo. Não se identificou nenhum medicamento com especificação explícita de indicação para neonatos. As faixas etárias com maior proporção de indicação pediátrica foram pré-escolar, escolar e adolescente (Tabela 2).

Tabela 2. Faixa etária dos 46 medicamentos com registro de indicação pediátrica no exterior e faixa etária dos 37 medicamentos com registro de indicação pediátrica no Brasil

\begin{tabular}{lcc} 
Faixa etária & $\begin{array}{c}\text { Exterior } \\
\mathbf{n = 4 6} \\
\mathbf{n}(\%)\end{array}$ & $\begin{array}{c}\text { Brasil } \\
\mathbf{n = 3 7} \\
\mathbf{n}(\%)\end{array}$ \\
\hline Estratificação & & \\
Recém-nascido prematuro & $3(6,5)$ & $4(10,8)$ \\
Recém-nascido a termo & $3(6,5)$ & $4(10,8)$ \\
Lactente & $16(34,8)$ & $8(21,6)$ \\
Pré-escolar & $27(58,7)$ & $21(56,7)$ \\
Escolar & $37(80,4)$ & $30(81,1)$ \\
Adolescente & $46(100,0)$ & $37(100,0)$ \\
\hline
\end{tabular}

Entre os 46 medicamentos com indicação pediátrica, um era comercializado em duas diferentes especialidades farmacêuticas, uma para uso nasal e outra para uso pulmonar. Considerando 47 especialidades farmacêuticas, verificou-se que $22(46,8 \%)$ destinavam-se à via oral, $12(25,5 \%)$ à endovenosa, $7(14,9 \%)$ à subcutânea, $2(4,3 \%)$ à nasal, 2 (4,3\%) à oftálmica, 1 (2,1\%) à dérmica e 1 (2,1\%) à pulmonar.

Em relação à forma farmacêutica das 47 especialidades farmacêuticas, as mais prevalentes foram as sólidas de uso oral $(42,5 \%)$ e parenterais $(40,4 \%)$, conforme apresentado na tabela 3. Entre as 20 especialidades farmacêuticas que eram formas sólidas, evidenciou-se predomínio de comprimidos e cápsulas, sendo que constavam apenas um comprimido dispersível e um mastigável. Foram observados duas especialidades em formas líquidas de uso oral.

Tabela 3. Forma farmacêutica dos 46 medicamentos com registro de indicação pediátrica no exterior e comercializados no Brasil de 2003 a 2013

\begin{tabular}{lc}
\hline Forma farmacêutica & $\mathbf{n}(\%)$ \\
\hline Formas farmacêuticas sólidas & $20(42,5)$ \\
Comprimido & $12(25,5)$ \\
Cápsula & $6(12,8)$ \\
Comprimido mastigável & $1(2,1)$ \\
Comprimido dispersível & $1(2,1)$ \\
\hline Formas parenterais & $19(40,4)$ \\
Parenteral endovenoso & $12(25,5)$ \\
Parenteral subcutâneo & $7(14,9)$ \\
Formas em aerossol* & $3(6,4)$ \\
Dispositivo de pó seco para inalação & $1(2,1)$ \\
Spray nasal & $2(4,3)$ \\
Formas líquidas de uso oral & $2(4,3)$ \\
Solução oftálmica & $2(4,3)$ \\
Pomada & $1(2,1)$ \\
\hline Total & $47(100,0)$ \\
\hline *Um medicamento apresenta duas formas farmacêuticas diferentes.
\end{tabular}

A adequação devido à capacidade do medicamento fornecer a dose recomendada foi superior a $80 \%$ em todas as faixas etárias (Figura 1). Em relação à adequação da forma farmacêutica, identificou-se que quanto maior a faixa etária, maior a proporção de adequação para uso pediátrico. Entre os 20 medicamentos em formas farmacêuticas sólidas, $10(50,0 \%)$ foram classificados como adequados para todas as faixas etárias pediátricas para os quais estavam registrados. Na avaliação da capacidade de fornecer a dose recomendada, identificou-se que $16(80,0 \%)$ eram adequados; as quatro $(20 \%)$ inadequações foram medicamentos cujo esquema posológico era 


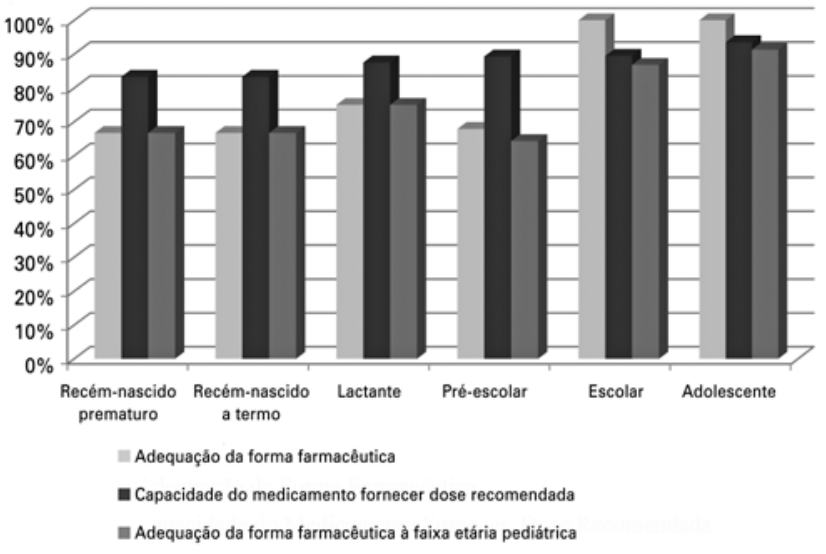

Figura 1. Adequação da forma farmacêutica dos 46 medicamentos com indicação pediátrica

prescrito em $\mathrm{mg} / \mathrm{kg} / \mathrm{dia}$. A taxa de adequação dos medicamentos comercializados nas formas farmacêuticas parenterais, líquidas de uso oral, tópicas e inalatórias foi de $100 \%$ nos dois critérios analisados. Na análise da adequação dos medicamentos, a faixa etária pediátrica apresentada na figura 1 mostra que a adequação foi menor para pré-escolares $(64,3 \%)$, recém-nascidos prematuros $(66,7 \%)$ e recém-nascidos a termo $(66,7 \%)$.

Os excipientes farmacêuticos com potencial de causar danos em crianças foram identificados em 22 $(46,8 \%)$ das 47 especialidades farmacêuticas, sendo que, em 3 especialidades, identificou-se mais de um excipiente. Entre as 25 ocorrências de excipientes, 12 $(48,0 \%)$ corresponderam a polisorbato $80,7(28 \%)$ a lactose, $3(12 \%)$ a cloreto de benzalcônio, $2(8,0 \%)$ a propilenoglicol e $1(4,0 \%)$ a etanol. Dentre as 22 especialidades que apresentaram excipientes com potencial para causar danos em crianças, $9(40,9 \%)$ eram de uso parenteral, $8(36,4 \%)$ sólidas de uso oral e $5(22,7 \%)$ abrangeram as demais formas farmacêuticas.

\section{DISCUSSÃO}

No período de 2003 a 2013, o número de medicamentos novos registrados no Brasil para a população pediátrica foi pequeno, uma vez que cerca de um terço dos medicamentos registrados para crianças menores de 6 anos foi classificado como inadequado para a faixa etária pediátrica a que se destinava. Além disso, verificou-se que quanto menor a idade da criança, menor o número de medicamentos novos pediátricos registrados no país. Estas características do mercado brasileiro de medicamentos é semelhante a descrita nos Estados Unidos e Europa. ${ }^{(8,25,26)}$ Verifica-se, assim, a existência de uma lacuna para conseguir a equidade na disponibilização de medicamentos destinados ao uso pediátrico, na mesma proporção em que são oferecidos para o uso adulto, atualmente, no mercado. Isto pode ser atribuído à complexidade de formular medicamentos para crianças que contemplem as especificidades das diferentes fases da infância. ${ }^{(1,26)}$ Percebe-se que as crianças ainda ocupam o status de órfãos terapêuticos, principalmente nas faixas etárias menores, pois há um deficit de medicamentos pediátricos. Apesar de as indústrias farmacêuticas terem promovido grandes avanços sobre o tema, aliados às recentes alterações na legislação, realizadas principalmente pelas agências reguladoras dos Estados Unidos e União Europeia, visando ampliar a disponibilidade de medicamentos adequados para crianças, ainda persiste a situação de órfãos terapêuticos. ${ }^{(7)} \mathrm{A}$ falta de lançamento, no período, de medicamentos para neonatologia, além do reduzido número de medicamentos para câncer pediátrico e para o tratamento de infecções bacterianas, torna o fato preocupante e mostra que os critérios das indústrias farmacêuticas para lançamentos de medicamentos não consideram integralmente as necessidades das crianças. ${ }^{(26)}$

Este deficit de medicamentos com indicação pediátrica é uma realidade mundial, não sendo diferente no Brasil; ${ }^{(12,27)}$ pode, consequentemente, trazer ameaças à segurança do paciente, por propiciar risco aumentado de eventos adversos, devido à utilização de medicamentos off label ou não licenciados. ${ }^{(27)}$

As formas farmacêuticas líquidas são consideradas pelos profissionais de saúde as mais adequadas para o tratamento das crianças, por apresentarem facilidade de administração e deglutição, e maior flexibilidade para ajustes de doses terapêuticas, além de permitirem melhor adesão das crianças ao tratamento. ${ }^{(5)}$ Apesar disto, as formulações líquidas orais apresentam problemas de palatabilidade, estabilidade química, física e/ou microbiológica e riscos de erros na medição da dose, o que se faz um desafio para o desenvolvimento de medicamentos pediátricos. ${ }^{(1,10)}$ Este desafio pode explicar o reduzido número destas formulações entre os medicamentos estudados.

Diante dos desafios para desenvolvimento de formas farmacêuticas líquidas adequadas às crianças, a OMS propôs, em 2008, que as formas sólidas flexíveis fossem consideradas as formulações preferidas para crianças. Elas correspondem às formas sólidas que não precisam ser ingeridas inteiras, como, por exemplo, os comprimidos dispersíveis, os efervescentes e os orodispersíveis. Para o maior acesso das crianças às formas sólidas flexíveis, tanto nos países desenvolvidos e em desenvolvimento, como nos países pobres, a OMS fomenta a implantação de plataformas tecnológicas para 
pesquisa e produção destes medicamentos. Apesar das vantagens técnicas e econômicas, além da facilidade de administração, a aceitação destas formulações pode ser influenciada por fatores culturais. Nesse sentido, a OMS busca conscientizar os pais e os cuidadores de crianças sobre os benefícios das formas flexíveis. Outro aspecto que visa aprimorar a aceitação pelas crianças e que está sendo abordado é a melhoria da palatabilidade das formulações. ${ }^{(28)}$

Entre os medicamentos novos registrados no período estudado, a proporção de medicamentos em formas sólidas flexíveis foi muito reduzida, mostrando a relevância das ações da OMS para ampliar o acesso a essas formas farmacêuticas. As formas sólidas foram prevalentes na coorte investigada e contribuíram significativamente para a classificação dos medicamentos como inadequados à faixa etária pediátrica, pois apenas uma apresentava capacidade de flexibilizar a dose, por ser um comprimido dispersível. Vale destacar que os esquemas posológicos mais frequentes para os medicamentos sólidos estudados eram em número de unidades da forma sólida a serem administradas. Se medicamentos com doses expressas em $\mathrm{mg} / \mathrm{kg} / \mathrm{dia}$ fossem em número maior, teria sido encontrada uma maior proporção de inadequação.

A fim de disponibilizar medicamentos de uso oral seguros e efetivos para as crianças, estão sendo propostas inovações tecnológicas, como minicomprimidos, películas orodispersíveis e formulações líquidas baseadas em leite. ${ }^{(10)}$ Os estudos com minicomprimidos estão em fase avançada, e os resultados mostraram que os de dimensão de $2 \mathrm{~mm}$ podem ser administrados a lactentes e neonatos prematuros; os de $4 \mathrm{~mm}$ são adequados para maiores de um ano. ${ }^{(10,11)}$ A aceitação dos minicomprimidos pelas crianças foi superior quando comparada com a aceitação de pó de uso oral, xarope, suspensão e solução. ${ }^{(10)}$ Outra vantagem dos minicomprimidos é a possibilidade de delineamento de formas farmacêuticas de liberação modificada, ${ }^{(1-10)}$ o que pode reduzir o número de doses administradas e facilitar a adesão. As diretrizes da EMA, que norteiam o desenvolvimento de medicamentos, estabelecem que o minicomprimido pode ser considerado medida para aumentar a aceitabilidade e a flexibilidade de doses para crianças. ${ }^{(10)}$

Os excipientes farmacêuticos, reconhecidos por serem desprovidos de ação farmacológica, constituem outro fator importante a ser considerado no desenvolvimento de medicamentos para crianças. A influência dos excipientes no perfil de segurança do medicamento pediátrico, devido à imaturidade de órgãos e sistemas corporais da criança, tem sido descrita. Excipientes que são seguros para adultos podem não ser seguros para crianças de idades menores. ${ }^{(1,10)}$ É crescente a preocu- pação com a presença de excipientes em medicamentos pediátricos, como o polisorbato 80 , que apresentou frequência alta dentre os medicamentos estudados..$^{(10,21-23)}$ Apesar disso, somente para neonatos, existe uma definição mais clara dos excipientes com maior potencial de causar dano. ${ }^{(23,29)}$

A influência de polisorbato 80 sobre a atividade da glicoproteína $\mathrm{P}$ e o potencial de induzir trombocitopenia, disfunção renal e acidose metabólica desperta atenção para os riscos na utilização de medicamentos pediátricos, especialmente em neonatos e crianças menores. ${ }^{(24)}$

Os excipientes selecionados por apresentarem potencial de causar danos em crianças foram encontrados em quase metade dos medicamentos estudados, o que demanda um alerta para a dimensão do problema. Entretanto, a avaliação da adequação do medicamento à faixa etária em relação aos excipientes farmacêuticos não pode ser considerada absoluta, uma vez que selecionou-se um limitado número de excipientes; a avaliação da segurança de um excipiente em uma formulação pediátrica depende do grupo etário alvo e de informações adicionais, como concentração e dose máxima permitida para ingestão diária. ${ }^{(10)}$ Estas informações não estão disponíveis nas bulas brasileiras. Devido a esses fatores, a avaliação da adequação do medicamento às faixas etárias pediátricas neste estudo abrangeu apenas a forma farmacêutica e a capacidade de fornecer a dose adequada.

O Brasil, diferente dos Estados Unidos e dos países da Europa, não possui legislação específica que regulamenta e incentiva o registro de medicamentos pediátricos. ${ }^{(12)}$ Entretanto, o Ministério da Saúde, diante das questões problemáticas que envolvem a utilização de medicamentos por crianças, instituiu um grupo de trabalho para incentivar a criação de políticas públicas direcionadas à melhoria da terapêutica farmacológica pediátrica. Em 2017, foi publicado o documento "Assistência Farmacêutica em Pediatria no Brasil Recomendações e estratégias para a ampliação da oferta do acesso e do uso racional de medicamentos em crianças", que apresenta ações abrangentes propostas pelo grupo de trabalho, para alterar o panorama nacional de dificuldades assistenciais às crianças e, consequentemente, melhorar a terapêutica pediátrica. ${ }^{(27)}$ Esta iniciativa do governo brasileiro está em consonância com o projeto Made Medicines for Children Size, da OMS, que incentiva os países a implantarem ações para ampliar a disponibilidade de medicamentos adequados para crianças. ${ }^{(30)}$

O presente estudo trouxe contribuições importantes para o conhecimento da adequação às faixas etárias pediátricas dos medicamentos novos registrados de 2003 a 2013 no Brasil. Além disso, é inovador, pois 
as pesquisas com medicamentos novos registrados no Brasil são anteriores a 2003 e não abordaram a perspectiva da criança. ${ }^{(27)}$ Todavia, é importante destacar, como limitações do estudo, que a avaliação do medicamento pediátrico restringiu-se aos aspectos farmacotécnicos. Outros aspectos, que visam contribuir para que os pais, cuidadores e profissionais de enfermagem administrem com segurança os medicamentos como, por exemplo, a natureza do dispositivo para medida das doses, a estabilidade da formulação e as instruções para utilização do medicamento não foram avaliados. A palatabilidade, que é importante para aceitação do medicamento pela criança e relevante para a promoção da adesão ao tratamento, também não foi avaliada. A ausência da avaliação destes fatores não permitiu conhecer a dimensão integral da adequação dos medicamentos ao uso pediátrico. Ademais, a análise foi restrita aos medicamentos cuja comercialização estava mantida em 2016, devido à necessidade de coletar informações relativas aos excipientes da formulação que constam na bula, mas não estão disponíveis na base de dados da ANVISA.

\section{CONCLUSÃo}

Os medicamentos destinados às crianças menores de 6 anos apresentaram menor frequência de adequação, considerando a forma farmacêutica e a capacidade de fornecer a dose recomendada. A disponibilidade e a proporção de adequação dos medicamentos para uso pediátrico aumentam com a elevação da faixa etária para a qual o medicamento é registrado. A frequência de medicamentos com presença de excipientes na formulação capazes de causar danos às crianças foi elevada.

\section{AGRADECIMENTOS}

À Fundação de Amparo à Pesquisa do Estado de Minas Gerais (FAPEMIG) e ao Conselho Nacional de Desenvolvimento Científico e Tecnológico (CNPQ), pelo convênio registrado no Sistema de Gestão de Convênios e Contratos de Repasse (SINCOV), número 794078/2013, e à Pró-Reitoria de Pesquisa da Universidade Federal de Minas Gerais.

\section{INFORMAÇÃO DOS AUTORES}

Castro JC: https://orcid.org/0000-0002-4654-2820

Botelho SF: https://orcid.org/0000-0002-6301-4683

Machado TR: https://orcid.org/0000-0002-1028-2369

Martins MA: https://orcid.org/0000-0002-5211-411X

Vieira LB: https://orcid.org/0000-0003-2273-5326

Reis AM: https://orcid.org/0000-0002-0017-7338

\section{REFERENCES}

1. Ivanovska V, Rademaker CM, van DijkL, Mantel-Teeuwisse AK. Pediatric drug formulations: a review of challenges and progress. Pediatrics. 2014; 134(2):361-72. Review.

2. Ernest TB, Elder DP, Martini LG, Roberts M, Ford JL. Developing paediatric medicines: identifying the needs and recognizing the challenges. J Pharm Pharmacol. 2007;59(8):1043-55. Review.

3. Walsh J. Reflection on the pharmaceutical formulation challenges associated with a paediatric investigation plan for an off-patent drug. AAPS PharmSciTech. 2017;18(2):250-6. Review.

4. Salunke S, Liu F, Batchelor H, Walsh J, Turner R, Ju TR, Tuleu C; European Paediatric Formulation Initiative (EuPFI). European Paediatric Formulation Initiative (EuPFI) - formulating ideas for better medicines for children. AAPS PharmSciTech. 2017;18(2):257-62. Review.

5. Liu F, Ranmal S, Batchelor HK, Orlu-Gul M, Ernest TB, Thomas IW, et al. Patient-centred pharmaceutical design to improve acceptability of medicines: similarities and differences in paediatric and geriatric populations. Drugs. 2014;74(16):1871-89. Review.

6. Stegemann S, Ternik RL, Onder G, Khan MA, van Riet-Nales DA. Defining patient centric pharmaceutical drug product design. AAPS J. 2016;18(5): 1047-55. Review.

7. Preis M, Breitkreutz J. Pediatric drug development and dosage form design. AAPS PharmSciTech. 2017;18(2):239-40.

8. Balakrishnan K, Grieve J, Tordoff J, Norris P, Reith D. Pediatric licensing status and the availability of suitable formulations for new medical entities approved in the United States between 1998 and 2002. J Clin Pharmacol. 2006;46(9):1038-43

9. Balakrishnan K, Tordoff $J$, Norris P, Reith D. Establishing a baseline for the monitoring of medicines availability for children in the UK: 1998-2002. Br J Clin Pharmacol. 2007;63(1):85-91

10. van Riet-Nales DA, Schobben AF, Vromans H, Egberts TC, Rademaker CM. Safe and effective pharmacotherapy in infants and preschool children: importance of formulation aspects. Arch Dis Child. 2016;101(7):662-9.

11. Van Riet-Nales DA, Kozarewicz P, Aylward B, de Vries R, Egberts TC, Rademaker CM, et al. Paediatric Drug Development and Formulation Design - a European Perspective. AAPS PharmSciTech. 2017;18(2):241-9. Review.

12. da Costa PQ, Rey LC, Coelho HL. Lack of drug preparations for use in children in Brazil. J Pediatr (Rio J). 2009;85(3):229-35.

13. Botelho SF, Martins MA, Vieira LB, Reis AM. Postmarketing safety events relating to new drugs approved in Brazil between 2003 and 2013: a retrospective cohort study. J Clin Pharmacol. 2017;57(4):493-9.

14. Agência Nacional de Vigilância Sanitária (ANVISA). Lista de preços de medicamentos [Internet]. Brasília (DF): ANVISA; 2016 [citado 2016 Jan 31]. Disponível em: http://portal.anvisa.gov.br/listas-de-precos

15. Agência Nacional de Vigilância Sanitária (ANVISA). Bulário eletrônico [Internet]. Brasília (DF): ANVISA; 2016 [citado 2016 Mar 31]. Disponível em: http://portal.anvisa.gov.br/bulario-eletronico1

16. WHO Collaborating Centre for Drug Statistics Methodology (WHO). ATC/ DDD Index 2018 [Internet]. Geneva: WHO; 2017 [Last updated: 2017-12-20], [cited 2018 July 20]. Available from: http://www.whocc.no/atc_ddd_index/

17. Williams K, Thomson D, Seto I, Contopoulos-loannidis DG, Ioannidis JP, Curtis S, Constantin E, Batmanabane G, Hartling L, Klassen T; StaR Child Health Group. Standard 6: age groups for pediatric trials. Pediatrics. 2012;129(Suppl 3): S153-60. Review.

18. Europe Medicines Agency (EMA). Pre-authorisation Evaluation of Medicines for Human Use. Committee for Medicinal Products for Human Use. Reflection paper: formulations of choice for the paediatric population [Internet]. London: EMA; 2006 [cited 2016 Feb 28]. Available from: http://www.ema.europa.eu/ docs/en_GB/document_library/Scientific_guideline/2009/09/WC500003782.pdf

19. Fontan JE, Mille F, Brion F, Aubin F, Ballereau F, Benoît G, Brunet ML, Braguier $D$, Combeau D, Dugast $P$, Gérout AC, May I, Meunier P, Naveau-Ploux C, Proust V, Samdjee F, Schlatter J, Thébault A, Vié M; Groupe Pédiatrie de la Société Française de Pharmacie Clinique. [Drug administration to paediatric inpatient]. Arch Pediatr. 2004;11(10):1173-84. French. 
20. World Health Organization (WHO). WHO Child Growth Standards: Methods and development. Length/height-for-age, weight-for-age, weight-for-length, weight-for-height and body mass index-for-age [Internet]. Geneva: WHO; 2006 [cited 2018 Apr 19]. Available from: http://www.who.int/childgrowth/ standards/Technical_report.pdf

21. Fabiano V, Mameli C, Zuccotti GV. Paediatric pharmacology: remember the excipients. Pharmacol Res. 2011;63(5):362-5.

22. Valeur KS, Hertel SA, Lundstrøm KE, Holst $H$. Safe excipient exposure in neonates and small children-protocol for the SEEN project. Dan Medical J. 2017;64(2). pii: A5324.

23. Nellis G, Metsvaht T, Varendi H, Toompere K, Lass J, Mesek I, Nunn AJ, Turner MA, Lutsar I; ESNEE consortium. Potentially harmful excipients in neonatal medicines: a pan-European observational study. Arch Dis Child. 2015;100(7):694-9.

24. Ursino MG, Poluzzi E, Caramella C, De Ponti F. Excipients in medicinal products used in gastroenterology as a possible cause of side effects. Regul Toxicol Pharmacol. 2011;60(1):93-105. Review.

25. Van Riet-Nales DA, de Jager KE, Schobben AF, Egberts TC, Rademaker C. The availability and age-appropriateness of medicines authorized for children in the Netherlands. Br J Clin Pharmacol. 2011;72(3):465-73.
26. Samiee-Zafarghandy S, Mazer-Amirshahi M, van den Anker JN. Trends in paediatric clinical pharmacology data in US pharmaceutical labelling. Arch Dis Child. 2014;99(9):862-5.

27. Brasil. Ministério da Saúde. Secretaria de Ciência, Tecnologia e Insumos Estratégicos. Departamento de Assistência Farmacêutica e Insumos Estratégicos. Assistência Farmacêutica em Pediatria no Brasil: recomendações e estratégias para a ampliação da oferta, do acesso e do Uso Racional de Medicamentos em crianças [Internet]. Brasília: Ministério da Saúde; 2017 [citado 2018 Abr 19]. Disponível em: http://bvsms.saude.gov.br/bvs/publicacoes/assistencia farmaceutica_pediatria_brasil_recomendacoes.pdf

28. Orubu ES, Tuleu C. Medicines for children: flexible solid oral formulations. Bull World Health Organ. 2017:95:238-40.

29. de Souza AS Jr, Dos Santos DB, Rey LC, Medeiros MG, Vieira MG, Coelho HL. Off-label use and harmful potential of drugs in a NICU in Brazil: a descriptive study. BMC Pediatrics. 2016;16:13.

30. Hoppu K, Anabwani G, Garcia-Bournissen F, Gazarian M, Kearns GL, Nakamura $\mathrm{H}$, et al. The status of paediatric medicines initiatives around the world--What has happened and what has not? Eur J Clin Pharmacol. 2012;68(1):1-10. 